\title{
NGHIÊN CỨU CƠ BẢN VỀ THỦY ÂM VÀ MÔ PHỎNG ĐÁNH GIÁ CÁC THAM SỐ CHÍNH
}

\author{
Phan Thanh Minh ${ }^{a^{*}}$, Nguyễn Ngọc Bình ${ }^{\mathrm{a}}$ \\ ${ }^{a}$ Khoa Nghiệp vu Viễn Thông, Truờng Đại học Thông tin Liên lạc, Khánh Hòa, Việt Nam \\ "Tác giả liên hệ: Email: phanthanhminh@tcu.edu.vn \\ Lịch sử bài báo \\ Nhận ngày 22 tháng 01 năm 2018 \\ Chỉnh sửa ngày 13 tháng 05 năm 2018 | Chấp nhận đăng ngày 14 tháng 05 năm 2018
}

\section{Tóm tắt}

Thông tin dưới nước cùng với các ứng dụng của nó là một lĩnh vục nghiên cứu đã và đang được phát triển nhanh chóng, mở rộng trong nhiều lĩnh vục nhu: Điều khiển xa trong ngành công nghiệp khai thác dầu mỏ ở ngoài biển; Tính toán ô nhiếm môi truờng trong các hệ thống thuộc về môi truờng; Truyền tiếng nói giữa các người nhái; Vẽ đáy đại duơng để tìm ra các nguồn tài nguyên mới; Thông tin liên lạc giữa các thiết bị ngầm;... Có hai cách thiết lạp việc trao đổi thông tin giữa các thiết bị dưới nước: Cách thứ nhất là kết nối bằng cáp giữa máy phát và máy thu, cách này bảo đảm chất lượng tín hiệu tốt và giảm thiểu nhũng tác động không mong muốn của môi truờng. Tuy nhiên, chi phí cho việc triển khai bảo đảm liên lạc cao, công tác bảo quản và bảo duỡng khó khăn, đặc biệt nếu việc trao đổi thông tin diễn ra ở độ sâu lón, trong điều kiện co động thì đảm bảo thông tin theo kiểu này rất phức tạp. Cách thứ hai là thiết lập thông tin giữa các thiết bị bằng cách sủ dụng nước nhu một môi trương truyền dẫn tín hiệu và kênh thông tin vô tuyến dưới nước nhu vậy được gọi là kênh thủy âm. Bài báo nghiên cúu nhũng vấn đề co bản để thực hiện truyền tin dưới nước bằng thủy âm, trong đó trình bày cơ sở chung để thưc hiện thông tin duới nước, mô phỏng hẹ thống thông tin thủy âm sủ dụng kỹ thuật điều chế QPSK (Quadrature Phase Shift Keying), kết luận đánh giá và đề xuất hướng nghiên cứu tiếp theo.

Từ khóa: QPSK; Thủy âm; Truyền dẫn.

Mã số định danh bài báo: http://tckh.dlu.edu.vn/index.php/tckhdhdl/article/view/423

Loại bài báo: Bài báo nghiên cứu gốc có bình duyệt

Bản quyền (C) 2018 (Các) Tác giả.

Cấp phép: Bài báo này được cấp phép theo CC BY-NC-ND 4.0 


\title{
RESEARCH ON UNDERWATER COMMUNICATION AND SIMULATED ASSESSMENT OF MAIN PARAMETERS
}

\author{
Phan Thanh Minh ${ }^{\mathrm{a}}$, Nguyen Ngoc Binh ${ }^{\mathrm{a}}$ \\ ${ }^{a}$ The Faculty of Professional Telecommunications, Telecommunications University, Khanhhoa, Vietnam \\ "Corresponding author: Email: phanthanhminh@tcu.edu.vn
}

\section{Article history}

Received: January $22^{\text {nd }}, 2018$

Received in revised form: May $13^{\text {th }}, 2018 \mid$ Accepted: May $14^{\text {th }}, 2018$

\begin{abstract}
Underwater communication and its applications is a research field that has been developing rapidly, extending in many fields such as remote control in the offshore oil industry, calculating environmental pollution, transmitting voice among frogmen, drawing the ocean floor to find new resources, communication between underground devices, etc. There are two ways of establishing information exchange between underwater devices. The simplest and most effective way is using a cable connection between the transmitter and the receiver, which ensures high quality of signal and minimizes unwanted effects of the environment. However, it has disadvantages such as high cost of deploying communication, difficult maintenance, and especially, if information exchanges take place at great depths and in mobile cases it would be very complex to ensure such an exchange. The second way is establishing information between devices using water as a signal transmission medium, which is called underwater communication channel. This article focuses on the basics of underwater communications including the general basis for conducting underwater communications, simulation of an underwater information system using QPSK modulation technique, and conclusions and recommendations for further researches.
\end{abstract}

Keywords: QPSK modulation technique; Signal transmission; Underwater acoustic.

Article identifier: http://tckh.dlu.edu.vn/index.php/tckhdhdl/article/view/423

Article type: (peer-reviewed) Full-length research article

Copyright (C) 2018 The author(s).

Licensing: This article is licensed under a CC BY-NC-ND 4.0 


\section{CỞ SỞ CHUNG ĐỂ THỰC HIỆN THÔNG TIN DƯớI NƯớC}

\subsection{Tại sao lại dùng âm thanh để truyền tin dưới nước?}

Bảng thống kê các tham số trong Bảng 1 cho thấy rằng sóng âm là tín hiệu thuận lợi nhất để truyền tin trong môi trường nước (suy hao truyền dẫn nhỏ, cự li liên lạc xa). Đại dương là một môi trường vô cùng phức tạp, tất cả đặc trưng riêng biệt của môi trường đại dương là tính tự nhiên không đồng nhất của nó. Có hai loại không đồng nhất: (i) Thông thường; và (ii) Ngẫu nhiên. Cùng với ảnh hưởng mạnh mẽ của trường sóng âm trong đại dương, sự thay đổi thông thường của tốc độ sóng âm so với các tham số của đại dương (nhiệt độ, độ sâu, độ mặn) đã dẫn tới hình thành "kênh sóng âm dưới nước" và điều đó là một hệ quả để truyền lan âm sóng đi xa. Sự không đồng nhất ngẫu nhiên của môi trường đại dương làm gia tăng sự tán xạ của sóng âm, do đó tạo ra những thay đổi thất thường trong trường sóng âm.

Bảng 1. So sánh các loại tín hiệu sóng âm, điện từ, quang trong môi trường nước

\begin{tabular}{llll}
\hline Tham số & Acoustic (Sóng âm) & Electromagnetic (Sóng điện từ) & Optical (Sóng quang) \\
\hline Nominal $(\mathrm{m} / \mathrm{s})$ & $\sim 1,500$ & $\sim 30,000,000$ & $\sim 30,000,000$ \\
Power Loss & $>0.1 \mathrm{~dB} / \mathrm{m} / \mathrm{Hz}$ & $\sim 28 \mathrm{~dB} / 1 \mathrm{Km} / 100 \mathrm{MHz}$ & $\alpha$ Turbidity \\
Bandwidth & $\mathrm{kHz}$ & $\mathrm{MHz}$ & $(10-150) \mathrm{MHz}$ \\
Frequency band & $\mathrm{kHz}$ & $\mathrm{MHz}$ & $\mathrm{THz}$ \\
Antenna size & $0.1 \mathrm{~m}$ & $0.5 \mathrm{~m}$ & $0.1 \mathrm{~m}$ \\
Effective range & $\mathrm{Km}$ & $10 \mathrm{~m}$ & $10-100 \mathrm{~m}$ \\
\hline
\end{tabular}

Nguồn: Tác giả tổng hợp từ Internet.

\section{2. Ảnh hưởng của đại dương đến truyền sóng âm dưới nước}

\subsubsection{Tốc độ âm thanh trong đại duơng}

Sự thay đổi của tốc độ âm thanh trong đại dương $c$ là tương đối nhỏ. Như là một qui luật, $c$ nằm giữa 1450 và $1540 \mathrm{~m} / \mathrm{s}$. Nhưng ngay cả các thay đổi nhỏ của $c$ cũng ảnh hưởng đáng kể tới sự truyền lan của âm thanh trong đại dương. Một số thí nghiệm và đo lường hiện nay chỉ ra rằng tốc độ âm thanh tăng một cách phức tạp khi nhiệt độ, áp suất thủy tĩnh (hoặc độ sâu) và lượng muối hòa tan trong nước tăng. Tốc độ âm thanh $c$ được đưa ra bởi Medwin và Clay (1998):

$$
c=1449.2+4.6 T-0.055 T^{2}+(1.34-0.01 T)(S-35)+0.016 z
$$

Ở đây nhiệt độ $T$ được tính bằng $\left[{ }^{\circ} C\right], S$ độ mặn $[\%]$, độ sâu $z$ tính bằng $[\mathrm{m}]$, tốc độ âm thanh tính bằng $[\mathrm{m} / \mathrm{s}]$. 


\subsubsection{Sự phu thuộc của tốc độ âm thanh vào nhiệt độ, độ mặn, độ sâu}

Dạng đặc trưng của tốc độ âm thanh là một hàm của $T, S$ và $z$ là:

$$
c(z)=c(T(z), S(z), z)
$$

Công thức (2) biểu thị mối quan hệ giữa $T, S, z$ và $c$ là quan trọng nhất đối với sự truyền lan của âm thanh trong đại dương. Đặc trưng của tốc độ âm thanh $c$ theo độ sâu $z$ là khác nhau đối với các vùng biển khác nhau, thay đổi theo thời gian (theo mùa). Tại các độ sâu dưới $1 \mathrm{~km}$, sự thay đổi của $T$ và $S$ thường nhỏ và tốc độ âm thanh tăng phần lớn do sự tăng của áp suất thủy tĩnh. Kết quả là tốc độ âm thanh tăng tuyến tính với độ sâu.

Hình 1 chỉ ra nét đặc trưng thông thường của nhiệt độ tại bề mặt của biển cao hơn tại đáy biển. Ở đây có thể thấy, nhiệt độ giảm so với độ sâu đến độ sâu $z=300 \mathrm{~m}$ và sau đó đạt giá trị không đổi. Điều này phù hợp với đặc trưng mùa hè của một vùng biển thông thường. Tốc độ âm thanh thay đổi cùng với sự thay đổi của nhiệt độ, độ mặn và độ sâu. Sự ảnh hưởng của nhiệt độ và áp suất đối với tốc độ âm thanh được chỉ ra trong Hình 2. Ở đây nó được phân thành ba vùng: Vùng thứ nhất nhiệt độ là nhân tố chi phối đến tốc độ âm thanh; Vùng thứ hai là vùng chuyển đổi có độ sâu từ 200-400m, cả nhiệt độ và độ sâu đều chi phối đến tốc độ âm thanh; và Vùng thứ ba trên $400 \mathrm{~m}$, tốc độ âm thanh hoàn toàn phụ thuộc vào độ sâu.

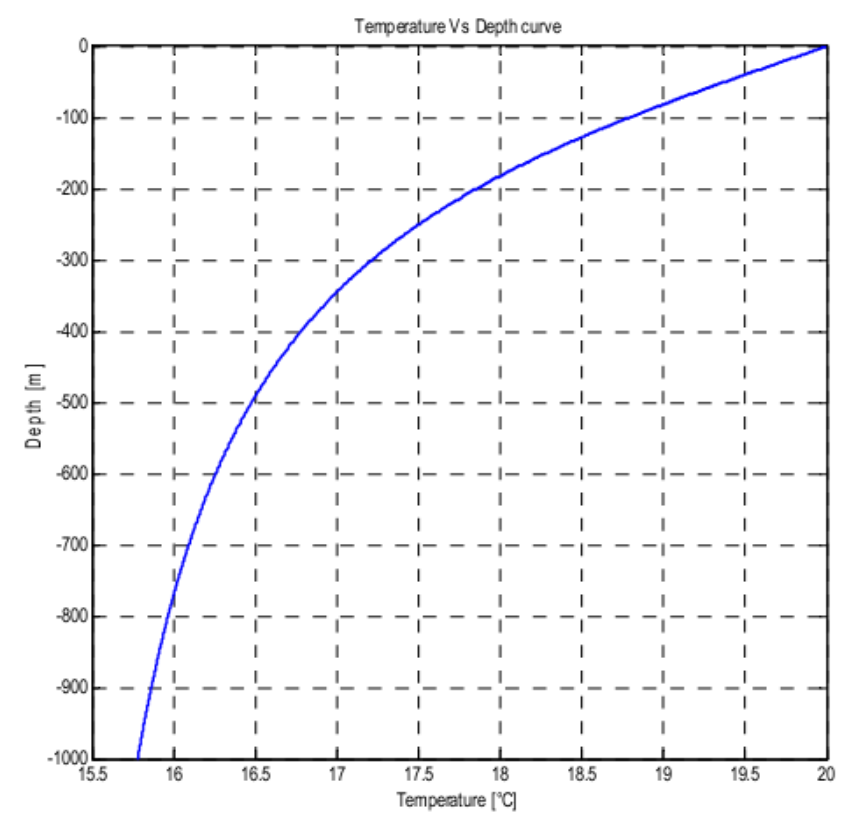

Hình 1. Nhiệt độ và độ sâu 


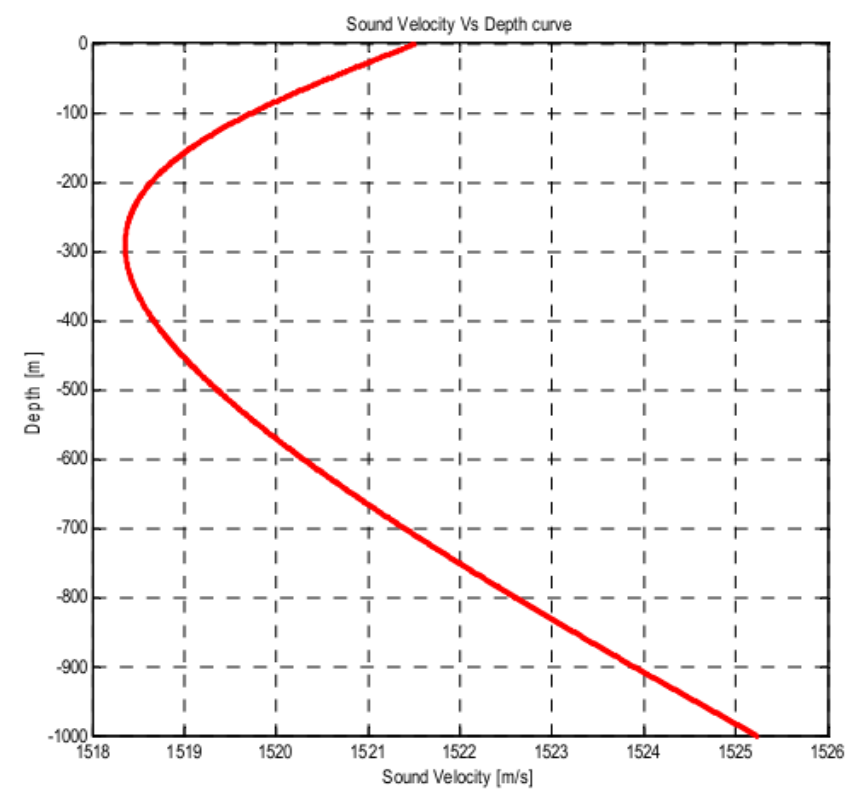

Hình 2. Tốc độ âm thanh và độ sâu

Sự phụ thuộc của $c$ vào độ mặn $S$ được chỉ ra trong Hình 3. Ở đây, với sự gia tăng của $S$, tốc độ âm thanh $c$ cũng tăng và giữ nguyên dạng đặc trưng của nó.

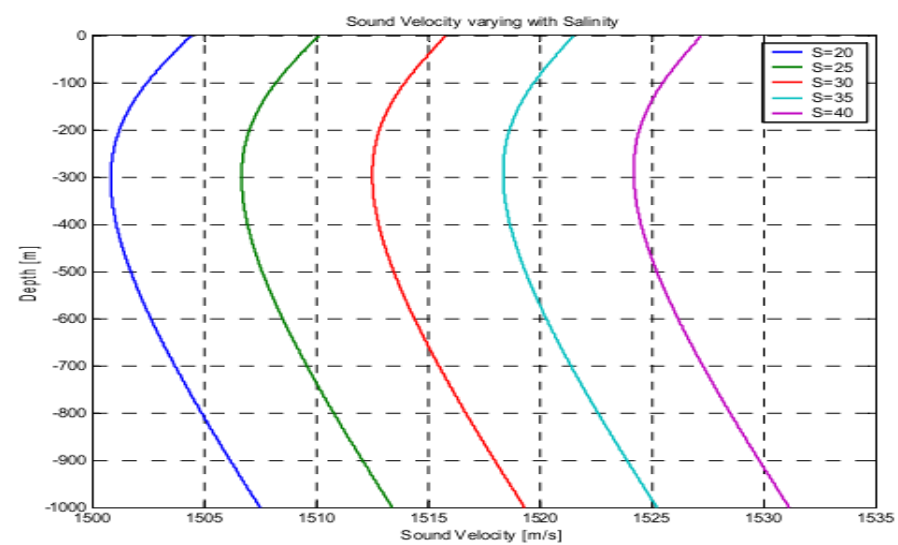

Hình 3. Tốc độ âm thanh và độ mặn

\subsubsection{Suy hao truyền dẫn của âm thanh trong môi trường nước}

- Tán xa hình cầu: Trong một môi trường đồng nhất và kéo dài vô tận, công suất phát ra bởi một nguồn được phát xạ theo tất cả các hướng trên bề mặt của một hình cầu. Điều này được gọi là phân bố hình cầu.

$$
G_{\text {sphere }}(r)=10 \log \left[\frac{I_{0}}{I}\right]_{\text {sphere }}=20 \log \left[\frac{r}{r_{0}}\right]^{2}
$$


Trong đó: $r o$ là khoảng cách tham khảo $(=1 \mathrm{~m})$; $I 0$ là cường độ âm thanh của nguồn tại khoảng cách $r o$; và $I$ là cường độ âm thanh của nguồn tại khoảng cách $r$.

- Tán xạ hình trụ: Tán xạ hình trụ tồn tại khi hai môi trường bị giới hạn bởi hai mặt phẳng phản xạ. Khoảng cách giữa các mặt phẳng được giả định là $h$ $>10 \lambda$. Trong đó, $\lambda$ biểu thị bước sóng của sóng âm.

$$
G_{c y l i n d e r}(r)=10 \log \left[\frac{I_{0}}{I}\right]_{c y l i n d e r}=10 \log \left[\frac{r}{r_{0}}\right]
$$

Năng lượng âm thanh của quá trình truyền sóng âm trong đại dương một phần bị hấp thụ do năng lượng bị chuyển thành nhiệt, một phần bị suy giảm do sóng âm bị tán xạ bởi sự không đồng nhất của môi trường. Nhưng trong các thí nghiệm ở đại dương thực, không thể phân biệt được giữa ảnh hưởng của sự hấp thụ và sự tán xạ. Cả hai hiện tượng trên góp phần làm suy giảm âm thanh trong nước biển.

Trên cơ sở những thí nghiệm rộng rãi và thực nghiệm, công thức thực nghiệm sau đây đối với hệ số suy giảm trong nước biển được đưa ra. Công thức này thỏa mãn khi tần số nằm trong miền (a) như Hình 4.

$$
\alpha=\frac{0.11 f^{2}}{1+f^{2}}+\frac{44 f^{2}}{4100+f^{2}}[\mathrm{~dB} / \mathrm{km}]
$$

Trong đó : $f$ là tần số $[k H z]$.

Theo Brekhovskikh và Lysanov (2003) thì công thức này thỏa mãn khi tần số nằm trong miền $(\mathrm{b})$ như Hình 4.

$$
\alpha=8.686 * 10^{3}\left(\frac{S \mathrm{~A} f_{T} f^{2}}{f_{T}^{2}}+\frac{B f^{2}}{f_{T}}\right)\left(1-6.54 * 10^{-4} P\right)[\mathrm{dB} / \mathrm{km}]
$$

Trong đó: $A=2.34 * 10^{-6}, B=3.38 * 10^{-6} ; S$ là độ mặn $[p p t] ; P$ là áp suất thủy tĩnh $\left[\mathrm{kg} / \mathrm{cm}^{2}\right] ; f$ là tần số $[\mathrm{kHz}]$; và $f_{T}=21.9 * 10^{6-1520 /(T+273)}[\mathrm{kHz}]$ là tần số nghỉ với $T$ là nhiệt độ tính bằng $\left[{ }^{0} \mathrm{C}\right]$, với dải nhiệt độ từ $0^{0}$ đến $30^{\circ} \mathrm{C}, f_{T}$ thay đổi xấp xỉ từ 59 đến 210 $k H z$. Công thức này thỏa mãn khi tần số nằm trong miền $(\mathrm{c})$ như Hình 4.

$$
\alpha=\underbrace{\frac{A_{1} P_{1} f_{1} f^{2}}{f_{1}^{2}+f^{2}}}_{B(O H)_{3}, \text { Boric acid }}+\underbrace{\frac{A_{2} P_{2} f_{2} f^{2}}{f_{2}^{2}+f^{2}}}_{M_{g} S O_{4}, \text { Magnesium sulphate }}+\underbrace{A_{3} P_{3} f^{2}}_{H_{2} O \text {, Pure water }}[\mathrm{dB} / \mathrm{km}]
$$

Trong đó: Boric axít $\mathrm{B}(\mathrm{OH})_{3}$ 


$$
A_{1}=\frac{8.686}{c} * 10^{0.78 p h-5} ; P_{1}=1 ; f_{1}=2.8 \sqrt{\frac{S}{35}} * 10^{4-\frac{1245}{T+273}}
$$

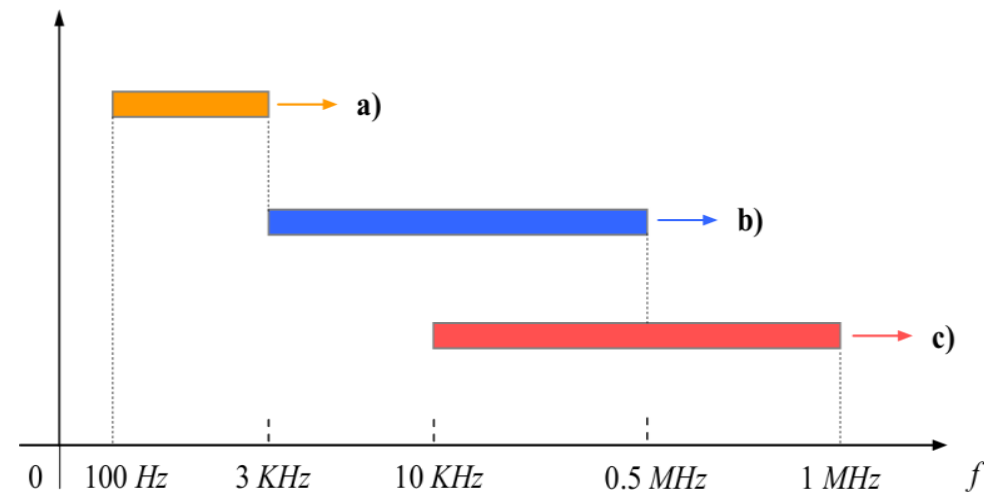

\section{Hình 4. Biểu đồ chỉ thị công thức thực nghiệm với các miền tần số khác nhau}

Magiê sunfat $\mathrm{MgSO}_{4}$

$$
A_{2}=21.44 * \frac{S}{c}(1+0.025 T) ; P_{2}=1-1.37 * 10^{-4} z_{\text {max }}+6.2 * 10^{-9} z_{\text {max }}^{2} ; f_{2}=\frac{8.17 * 10^{8-\frac{1990}{(T+273)}}}{1+0.0018(S-35)}
$$

Tốc độ âm thanh xấp xỉ được cho bởi:

$$
c=1412+3.21 T+1.19 S+0.0167 z_{\max }
$$

Trong các miền chuyển tiếp nó tương ứng với $f$. Sự suy giảm tăng tương ứng với sự tăng của độ mặn và nhiệt độ, Hình 5 thể hiện sự suy giảm tăng tương ứng với tần số tăng. Khi nhiệt độ tăng thì độ suy giảm tăng (Hình 6).

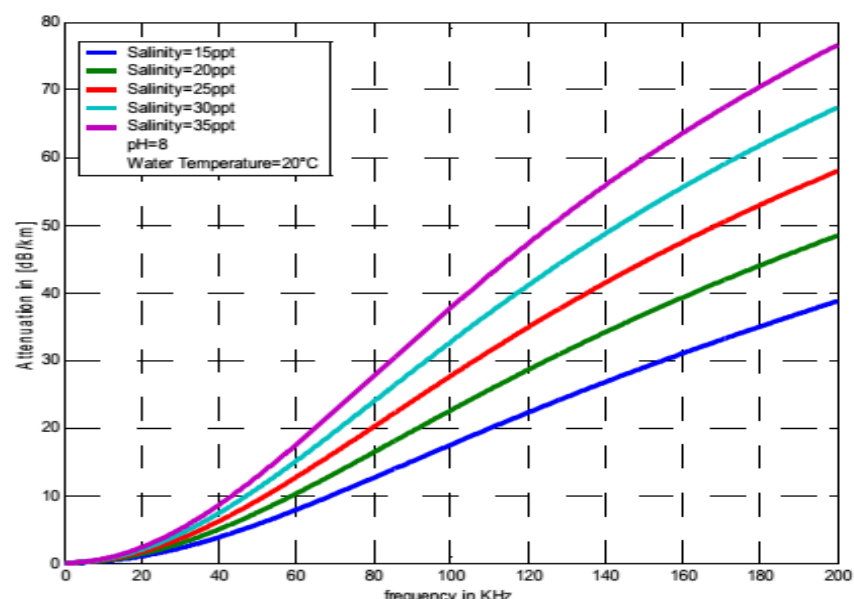

Hình 5. Hệ số suy giảm thay đổi theo độ mặn và nhiệt độ $20^{0} \mathrm{C}$ 


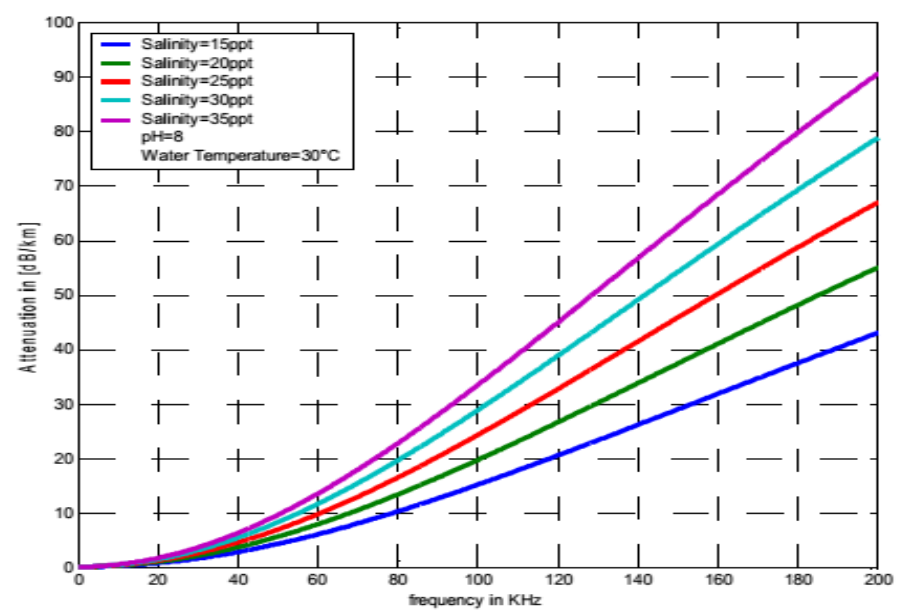

\section{Hình 6. Hệ số suy giảm thay đổi theo độ mặn và nhiệt độ $30^{0} \mathrm{C}$}

- Sự suy giảm của âm thanh trong lớp trầm tích: Sự suy giảm của âm thanh trong lớp trầm tích chủ yếu phụ thuộc vào sự thay đổi dạng của đáy. Cấu trúc của lớp đáy được tượng trưng bởi $b t$, biểu thị chất liệu lớp trầm tích của đại dương. Bảng 2 đưa ra các giá trị của $b t$ cho mỗi dạng của lớp trầm tích. Công thức thực nghiệm sau được đưa ra để tìm sự suy giảm của sóng âm trong lớp trầm tích phụ thuộc vào $b t$.

$\alpha_{s}=\frac{1}{8.686} K\left(\frac{f}{1 \mathrm{kHz}}\right)^{n}\left[\frac{1}{\mathrm{~m}}\right]$

Trong đó: $\alpha_{s}$ là sự suy giảm của sóng âm trong lớp trầm tích.

\section{Bảng 2. Các giá trị của $b t$ cho mỗi dạng của lớp trầm tích}

\begin{tabular}{ll}
\hline Dạng của lớp trầm tích & Giá trị bt \\
\hline Cát rất thô & 0 \\
Cát thô & 1 \\
Cát cỡ trung bình & 2 \\
Cát mịn & 3 \\
Cát rất mịn & 4 \\
Bùn rất thô & 5 \\
Bùn thô & 6 \\
Bùn cỡ trung bình & 7 \\
Bùn mịn & 8 \\
Bùn rất mịn & 9 \\
Đất sét & 10 \\
\hline
\end{tabular}


Bảng 3 đưa ra các giá trị của $K$ và $n$ đối với bốn dạng của lớp trầm tích.

Bảng 3. Các giá trị của $K$ và $n$ đối với bốn dạng của lớp trầm tích.

\begin{tabular}{lll}
\hline Dạng của lớp trầm tích & $K$ & $n$ \\
\hline Bùn rất mịn & 0.17 & 0.96 \\
Cát mịn & 0.45 & 1.02 \\
Cát cỡ trung bình & 0.48 & 0.98 \\
Cát thô & 0.53 & 0.96 \\
\hline
\end{tabular}

\subsubsection{Hiệu ứng Doppler}

Chuyển động tương đối giữa máy thu và máy phát gây nên thay đổi trong đáp ứng kênh truyền do hiệu ứng Doppler. Biên độ của hiệu ứng Doppler tỉ lệ với tỉ số $a=$ $v / c$, trong đó $v$ là chuyển động tương đối giữa máy thu và máy phát, $c$ là vận tốc âm thanh trong nước. Do vận tốc của âm thanh trong nước là khá nhỏ khi so sánh với vận tốc của sóng điện từ trên không trung nên ảnh hưởng của hiệu ứng Doppler là rất lớn. Các thiết bị tự động dưới nước di chuyển với tốc độ khoảng vài $\mathrm{m} / \mathrm{s}$, tuy nhiên kể cả khi không có những chuyển động có mục đích thì những ảnh hưởng như sự trôi dạt gây nên bởi sóng, thủy triều cũng luôn tồn tại. Nói cách khác luôn có chuyển động tương đối giữa máy thu và phát, hệ thống thông tin thủy âm cần được thiết kế nhằm giải quyết vấn đề này. Vấn đề này có nhiều điểm tương đồng với kênh vô tuyến truyền dẫn qua vệ tinh. Méo tín hiệu gây ra do chuyển động của thiết bị tác động đến việc thiết kế thuật toán đồng bộ và ước lượng kênh truyền.

Mức độ ảnh hưởng của méo lên tín hiệu phụ thuộc trực tiếp và giá trị của $a$. Làm phép so sánh như sau: Với hệ thống thông tin vô tuyến, với độ dịch chuyển tương đối giữa nguồn thu và phát là $160 \mathrm{~km} / \mathrm{h}$ thì $a=1.5 \times 10^{-7}$, giá trị này đủ nhỏ để ảnh hưởng của hiệu ứng Doppler có thể được bỏ qua. Nói cách khác, việc xem xét ảnh hưởng độ méo của tín hiệu trong quá trình đồng bộ là không cần thiết. Xác xuất lỗi bit là rất nhỏ. Ngược lại, với kênh thông tin thủy âm, giả sử nguồn phát và thu chịu sự dịch chuyển 0.5 $\mathrm{m} / \mathrm{s}$ ( $1 \mathrm{knot})$, thì giá trị của $a$ vào khoảng $a=3 \times 10^{-4}$. Nếu như dịch chuyển tương đối lên tới vài $\mathrm{m} / \mathrm{s}$ (tàu ngầm có thể dịch chuyển với vận tốc lớn hơn), giá trị của $a$ vào khoảng $a=10^{-3}$, và đây là giá trị không thể bỏ qua được.

Dịch chuyển Doppler và trải phổ Doppler sinh ra do chuyển động tương đối là một trong những yếu tố khác biệt giữa kênh thông tin thủy âm với kênh thông tin vô tuyến. Trải phổ Doppler gây lệch pha, trễ đồng bộ. Trong hệ thống thông tin thủy âm dùng đa sóng mang, hiệu ứng Doppler còn gây ra méo đặc biệt nghiêm trọng. Trong kênh thông tin vô tuyến trên không trung, sự nén, giãn theo thời gian là có thể bỏ qua, hiệu ứng Doppler là như nhau với tất cả các sóng mang con. Với kênh thông tin thủy 
âm, ảnh hưởng của dịch chuyển Doppler lên mỗi sóng mang con là khác nhau đáng kể, điều này gây nên méo Doppler không đồng bộ trên toàn bộ băng thông tín hiệu.

\section{MÔ PHỎNG HỆ THỐNG THÔNG TIN THỦY ÂM SỦ DỤNG KỸ THUẠTT ĐIỀU CHẾ QPSK}

\subsection{Sơ đồ khối hệ thống thông tin thủy âm}

Để tiến tới một hệ thống rời rạc theo thời gian mong muốn, xét hệ thống thông tin như Hình 7. Đây là hệ thống thông thường được thiết lập mà có thể đặc trưng cho bất kỳ hệ thống nào sử dụng kiểu điều chế biên độ cầu phương QAM (Quadrature Amplitude Modulation). Tóm tắt về hệ thống được mồ tả như sau: Tại phía phát, các ký hiệu tuần tự $d(n)$ được chuyển đổi sang tín hiệu băng gốc liên tục theo thời gian $s b b(t)$ bởi một bộ điều chế biên độ xung (Pulse Amplitude Modulation-PAM). Trong đó các giá trị $d(n)$ là các số phức rời rạc. Chuyển đổi tần số đường lên được thực hiện bằng cách nhân tín hiệu với $e^{j 2 \pi f_{c} t}$, kết quả là tín hiệu băng thông $s(t)$ được truyền qua kênh truyền như đã phân tích.

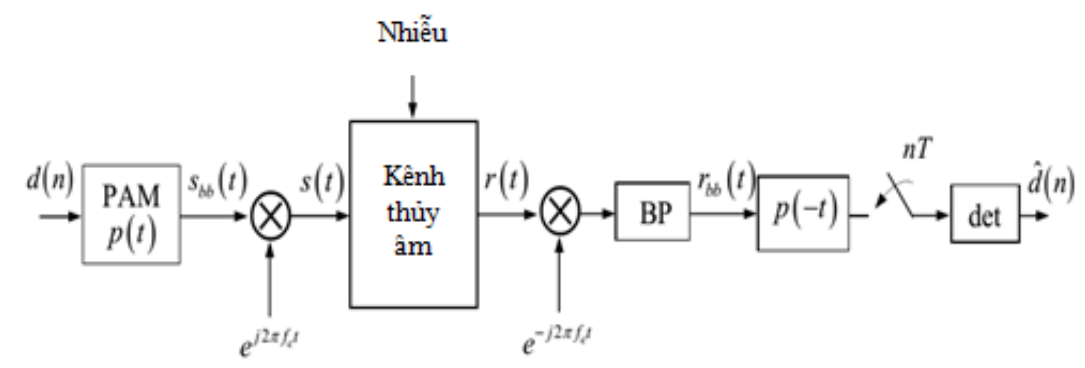

Hình 7. Hệ thống mô phỏng kênh thủy âm

Tại đầu thu, để tách sóng mang, tín hiệu thu $r(t)$ được xử lý bằng một bộ đổi tần xuống mà có tín hiệu đầu ra phù hợp với tín hiệu tương đương băng gốc $r_{b b}(t)$. Sau bộ chuyển đổi đường xuống là bộ lọc thông thấp và bộ lọc phối hợp. Bộ tách sóng thực hiện ước lượng các ký hiệu đã được phát. Như đề cập ở trên, các đặc trưng của tín hiệu băng gốc sẽ có nhiều thuận lợi cho việc mô phỏng hệ thống. Hình 8 tượng trưng cho hệ thống tương đương băng gốc.

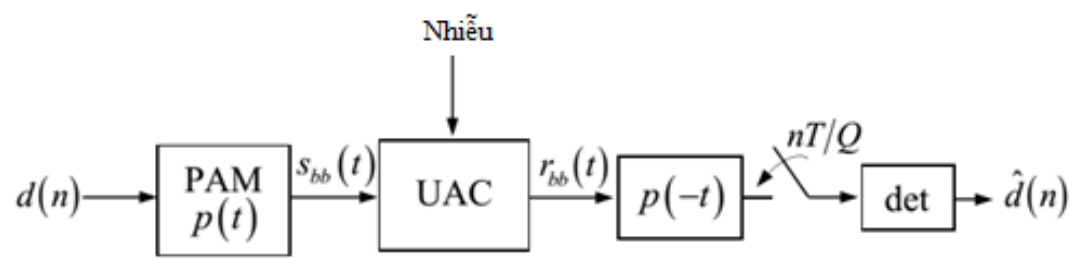

Hình 8. Hệ thống tương đương băng gốc 
Mô phỏng thường được dựa trên hệ thống oversampled, có nghĩa là tốc độ lấy mẫu cao hơn tốc độ ký hiệu. Nhìn chung tốc độ lấy mẫu cao hơn sẽ phản ảnh chính xác hơn hệ thống nguyên bản. Tuy nhiên điều này dẫn tới thời gian mô phỏng dài hơn do nhiều mẫu hơn cần phải xử lý. Nhìn chung để sử dụng tốc độ oversample, tốc độ này là bội số của tốc độ ký hiệu. Trong đó $Q$ là số bit trên một ký hiệu, $Q$ là một số tự nhiên.

\subsection{Xây dựng mô hình mô phỏng}

Hệ thống mô phỏng được minh họa trong Hình 9. Trong đó bao gồm một nguồn bit, máy phát, kênh truyền, máy thu và một bộ tính tỉ số lỗi bit. Nguồn bit tạo ra các bit nhị phân tuần tự ngẫu nhiên và nó được phát đi bởi máy phát. Thông thường một nguồn bit ngẫu nhiên được dùng trong các mô phỏng và được sử dụng trong mô phỏng của bài báo. Máy phát chuyển các bit sang ký hiệu QPSK, đưa qua bộ tạo dạng xung và chuyển đổi đường lên được thực hiện để đưa đến tần số sóng mang yêu cầu.

Tín hiệu đầu ra của máy phát được phát đi qua kênh thủy âm. Máy thu nhận tín hiệu này từ kênh truyền, ước lượng pha và định thời, giải điều chế các tín hiệu QPSK nhận được thành các bit tin và cung cấp cho khối tính toán tỉ số lỗi bit. Ở đây việc tính số lượng các bit lỗi xuất hiện để khảo sát hiệu năng của hệ thống.

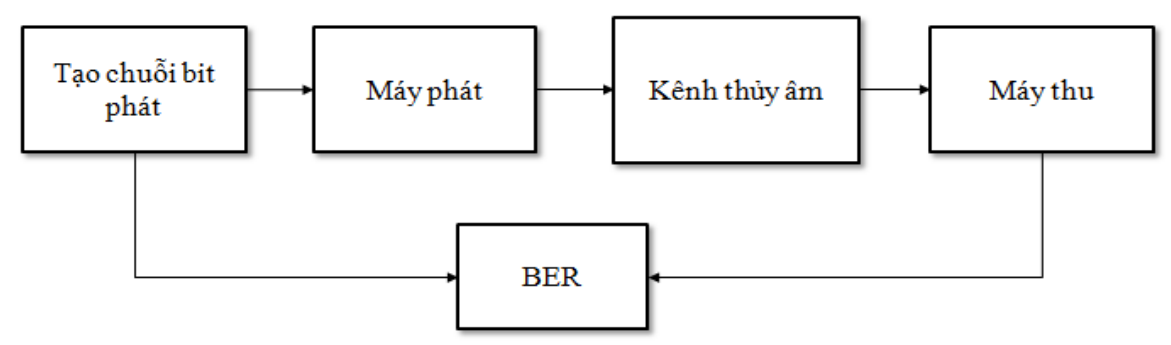

\section{Hình 9. Hệ thống mô phỏng}

\subsection{Kết quả mô phỏng}

Sử dụng lập trình Matlap, tiến hành đánh giá một số tham số như sau:

\subsubsection{Kết quả mô phỏng đối với các loại đáy}

Tiến hành đánh giá cho các loại đáy mà giá trị $b t$ được trình bày trong Bảng 2 . Các tham số khác được mô tả như sau: Vị trí nguồn $\left(r_{s}, z s\right)=(0,25) \mathrm{m}$; Vị trí máy thu $\left(r_{R_{1}}\right.$, $\left.z_{R_{1}}\right)=(500,25) \mathrm{m}$; Tốc độ âm thanh $c=1500 \mathrm{~m} / \mathrm{s}$; Độ sâu của nước $D=40 \mathrm{~m}$; Độ mặn $S=35$ ppt; Nhiệt độ của nước $T=14^{0} \mathrm{C}$; và Độ $p H$ là $p H=8$.

Nhận thấy rằng tín hiệu phản xạ từ đáy tới bên thu có biên độ rất khác nhau cho những môi trường khác nhau. Với môi trường mà đáy có tính chất phản xạ tốt, tín hiệu thu do sự phản xạ từ đáy đóng vai trò đáng kể. Như vậy, khi tiến hành triển khai hệ 
thống trên thực tế, việc nghiên cứu tính chất phản xạ của mặt đáy là vô cùng quan trọng. Sự ảnh hưởng của tính chất đa đường đến tín hiệu trong mồi trường đáy bùn và đáy đất sét hầu như không có sự khác biệt nào đáng kể.

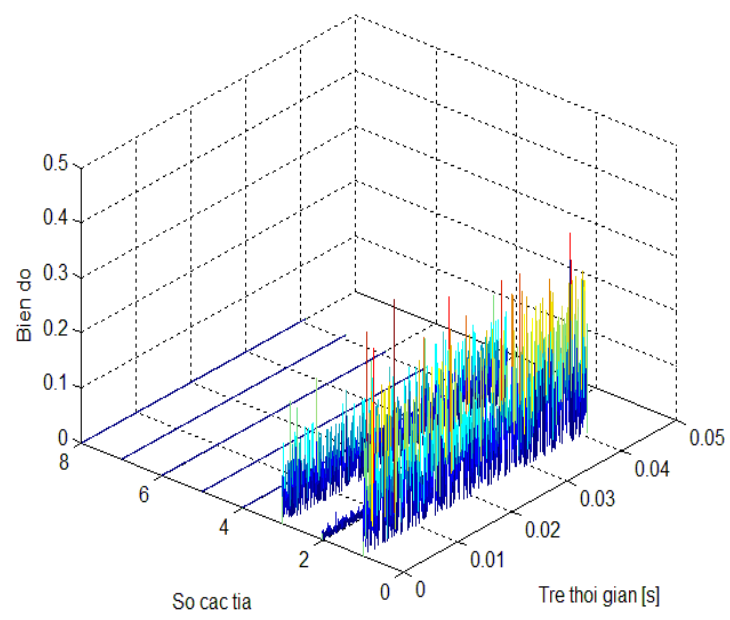

(a)

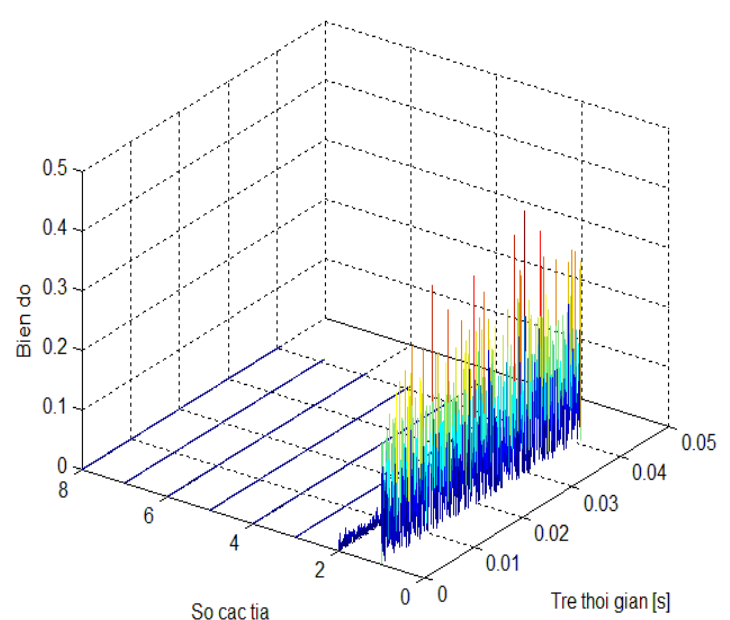

(b)

Hình 10. Tín hiệu đa đường trong môi trường đáy cát và đáy bùn

Ghi chú: a) Môi trường đáy là cát; b) Môi trường đáy là bùn.

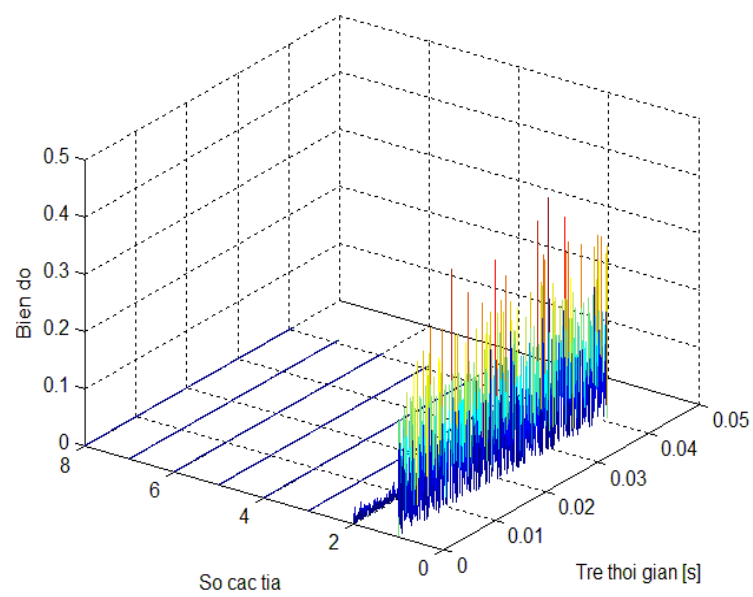

(a)

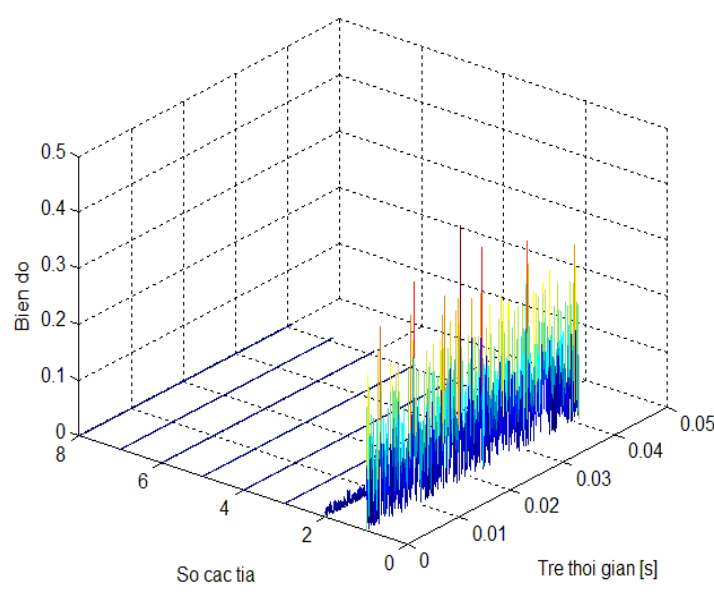

(b)

Hình 11. Tín hiệu đa đường trong môi trường đáy bùn và đáy là đất sét

Ghi chú: a) Môi trường đáy là bùn; b) Môi trường đáy là đất sét. 
thu thay đổi

2.3.2. Kết quả mô phỏng đối với truờng hợp khoảng cách giũa máy phát và máy

Tham số mô phỏng (Hình 12) bao gồm: Tần số sóng mang là $30 \mathrm{kHz}$; Dạng đáy $b t=1$; Vị trí nguồn $\left(r_{s}, z_{s_{1}}\right)=(0,20) \mathrm{m}$; Vị trí máy thu $\left(r_{R_{1}}, z_{R_{1}}\right)=(x, 20) \mathrm{m}$, với $x=10$, $500,1000,1500$.

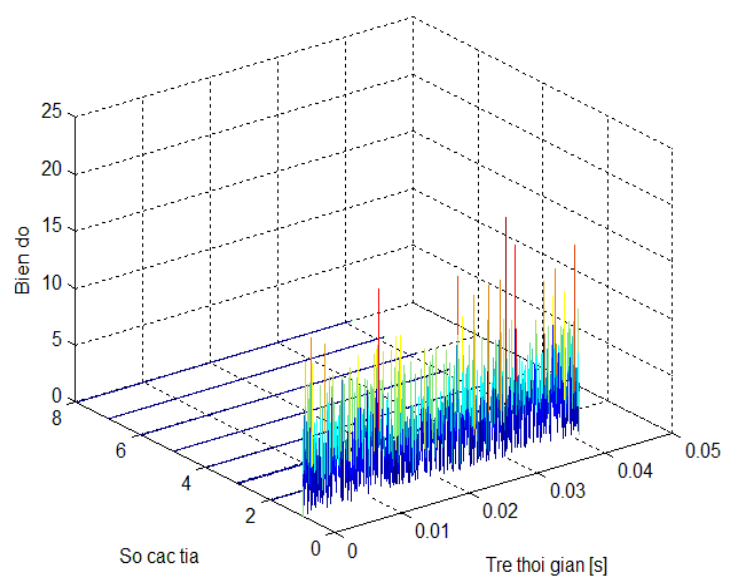

(a)

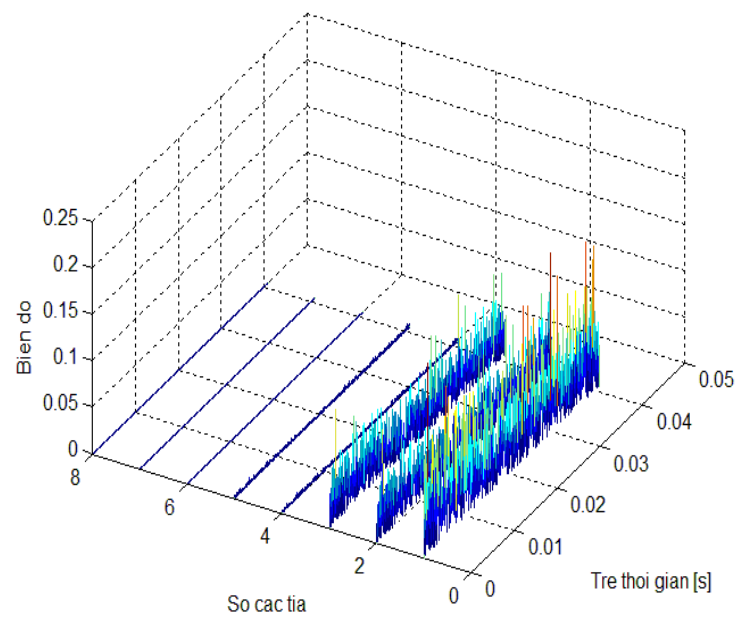

(c)

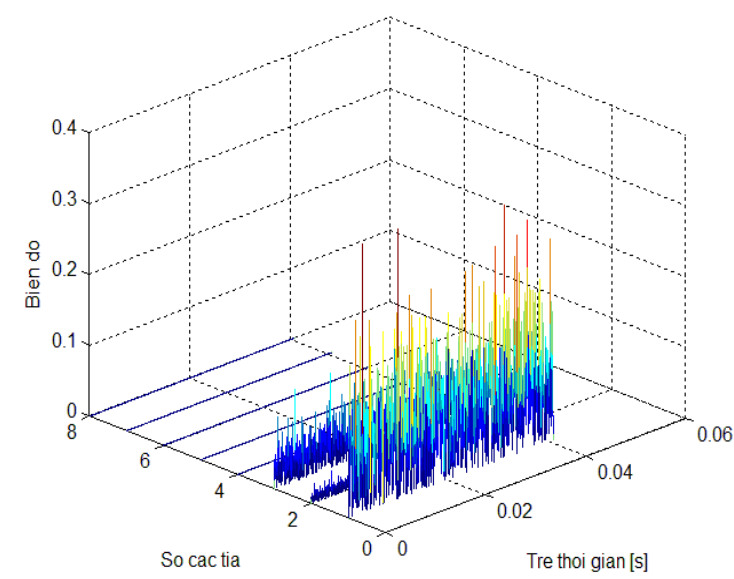

(b)

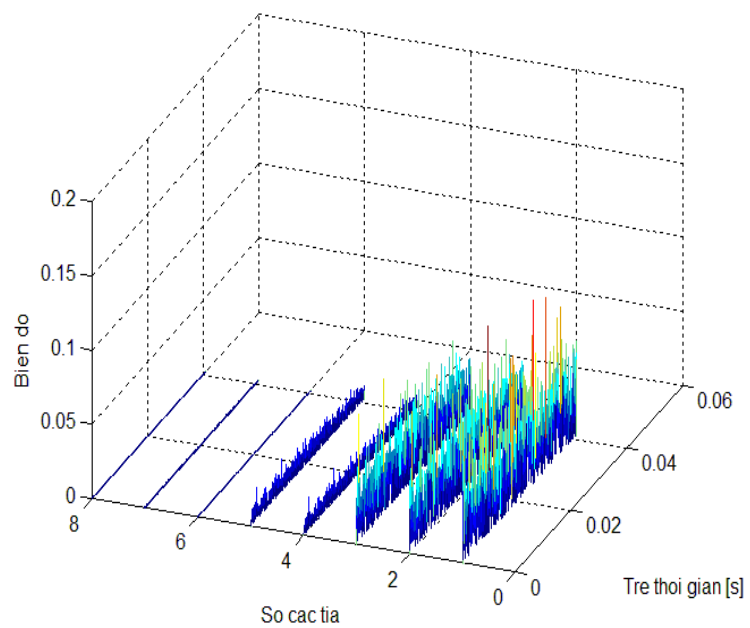

(d)

\section{Hình 12. Tính chất đa đường tại các khoảng cách khác nhau với đáy cát}

Ghi chú: a) $10 \mathrm{~m}$; b) $500 \mathrm{~m}$; c) 1000m; và d) $1500 \mathrm{~m}$.

\subsection{3. Đặc tuyến BER}

Trong thông tin, mục tiêu là phải thu được tỉ số tín hiệu trên tạp âm cao nhất. Đối với thông tin dưới nước thì nhiễu có hai dạng, một là nhiễu do môi trường xung quanh tạo nên và thứ hai là hiện tượng truyền dẫn đa đường của chính tín hiệu đó. Do 
vậy, chúng tôi xem xét ảnh hưởng của hiện tượng truyền dẫn đa đường của kênh thủy âm và tất cả các ảnh hưởng đối với kênh truyền này. Sau đây là một số kết quả mô phỏng chỉ ra tỉ số lỗi bit $(B E R)$ đối với trường hợp chỉ có đường trực tiếp và đa đường cho hai trường hợp khi cấu trúc của dạng đáy khác nhau và khoảng cách giữa máy phát và máy thu khác nhau.

\section{- Đặc tuyến $B E R$ khi chưa xét đến ảnh hưởng của đa đường}

Tham số mô phỏng: Tốc độ âm thanh $c=1500 \mathrm{~m} / \mathrm{s}$; Độ sâu của nước $D=40 \mathrm{~m}$; Độ mặn $S=35$ ppt; Nhiệt độ của nước $T=14^{0} \mathrm{C}$; Độ $p H=8$; Tốc độ gió $v_{w}=6 \mathrm{knots}$; Vị trí nguồn $\left(r_{s}, z s\right)=(0, x) \mathrm{m}, x=10.35$; Vị trí máy thu $\left(r_{R_{1}}, z_{R_{1}}\right)=(1000, x) \mathrm{m}$; và Dạng đáy $b t=$ bùn thô. Hình 13 biểu thị giá trị $B E R$ của đường trực tiếp. Trong trường hợp này, chúng tôi chỉ xét đến đường trực tiếp do đó không có bất cứ một nhiễu nào, vì vậy chỉ có sự suy giảm về độ lớn của tín hiệu nên ta có $B E R$ của đường trực tiếp bằng không.

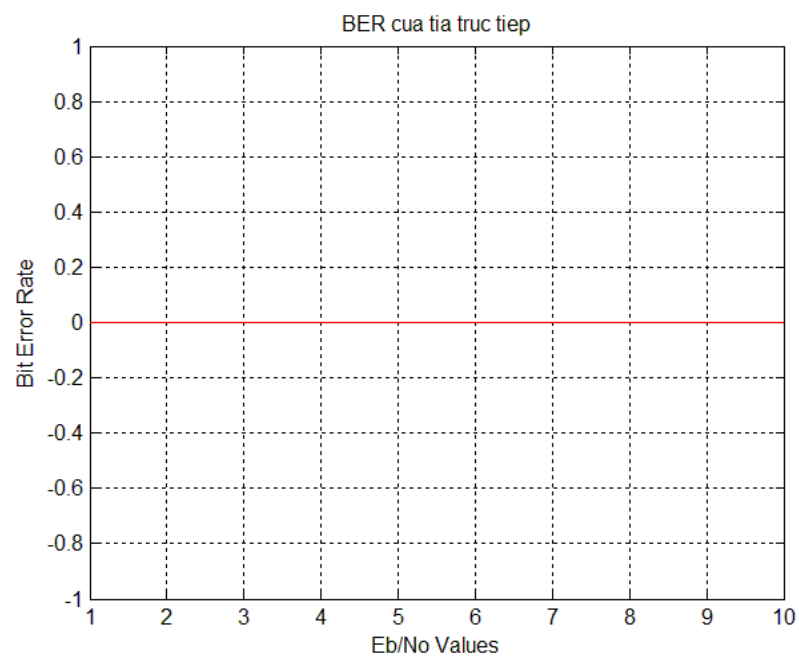

Hình 13. BER của tia trực tiếp

- Đặc tuyến $B E R$ khi xét đến ảnh hưởng của truyền dẫn đa đường

Xét hai trường hợp, trường hợp một dạng đáy là bùn và trường hợp thứ hai dạng đáy là cát khi có tác động của hiện tượng truyền dẫn đa đường. Tham số mô phỏng: Vị trí nguồn $\left(r_{s, z s}\right)=(0,10) \mathrm{m}$; Vị trí máy thu $\left(r_{R, z R}\right)=(500,35) \mathrm{m}$; Tốc độ âm thanh $c=1500 \mathrm{~m} / \mathrm{s}$; Độ sâu của nước $D=40 \mathrm{~m}$; Độ mặn $S=35 \mathrm{ppt}$; Nhiệt độ của nước $T=14^{0} \mathrm{C}$; Độ $p H=8$; Tốc độ gió $v_{\mathrm{w}}=6$ knots; và Dạng đáy: $b t=0,7$.

Hình 14 và Hình 15 cho thấy: Với môi trường có đáy phản xạ $b t=7$, thành phần phản xạ sẽ có biên độ nhỏ hơn so với trường hợp đáy có hệ số phản xạ là $b t=0$, do đó 
ảnh hưởng của thành phần phản xạ sẽ nhỏ hơn. Vì vậy, tỉ lệ lỗi bit của trường hợp $b t=7$ sẽ nhỏ hơn tỉ lệ lỗi bit của $b t=0$.

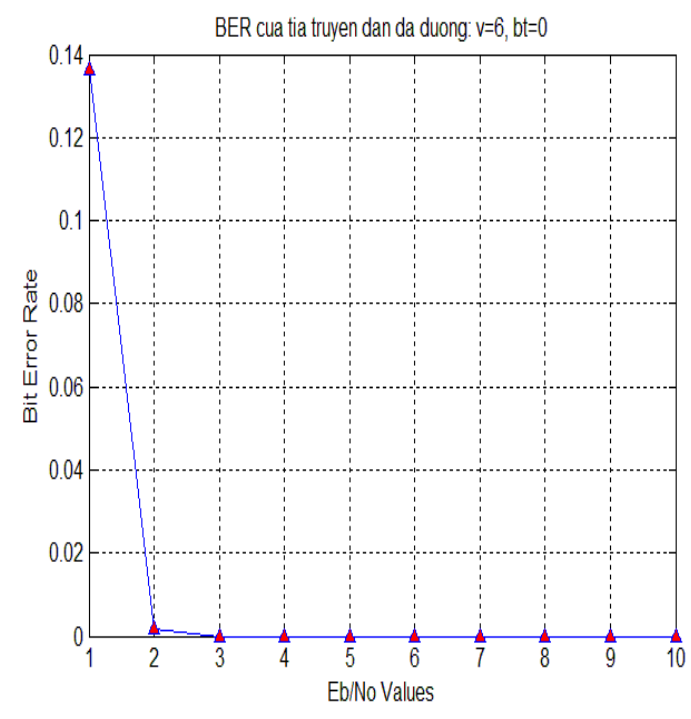

(a)

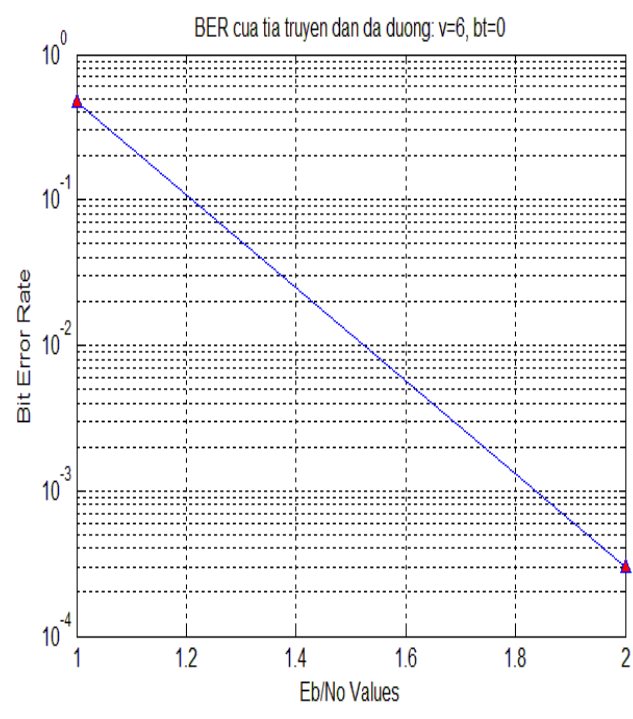

(b)

Hình 14. Biểu đồ BER đối với trường hợp $b t=0$

Ghi chú: a) Thang tuyến tính; b) Thang log.

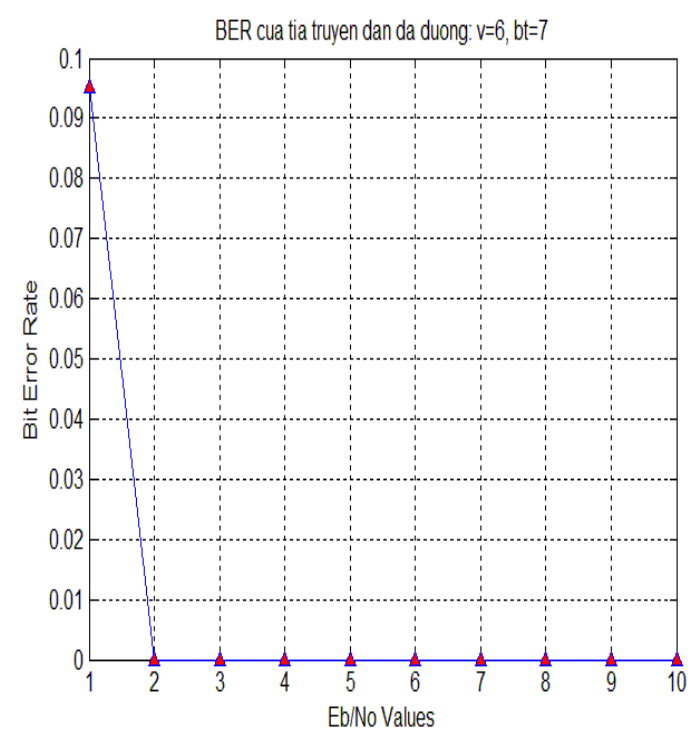

(a)

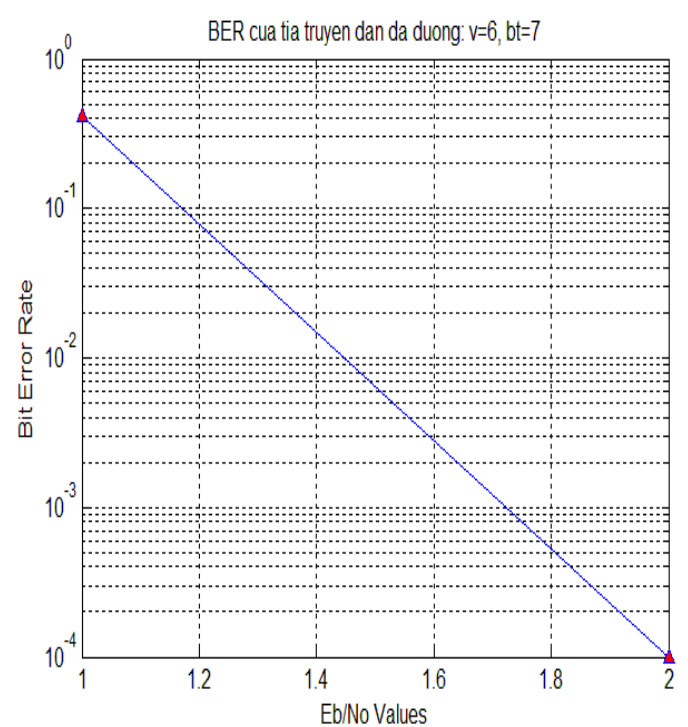

(b)

Hình 15. Biểu đồ BER đối với trường hợp $b t=7$

Ghi chú: a) Thang tuyến tính; b) Thang log. 


\section{KẾT LUẬN}

Thủy âm ngày càng được ứng dụng mạnh mẽ và đạt hiệu quả cao trong phát triển kinh tế cũng như bảo vệ chủ quyền lãnh thổ quốc gia. Những kết quả nghiên cứu trên của bài báo đã đưa ra kết quả về những tác động của các yếu tố đến quá trình truyền tin trong môi trường nước biển. Mô phỏng đưa ra kết quả cụ thể cho một mô hình hệ thống thông tin thủy âm sử dụng kỹ thuật điều chế QPSK. Kết quả có thể được ứng dụng trong việc chế tạo các thiết bị truyền tin dưới nước, trên cơ sở đó tổ chức xây dựng hệ thống thông tin trên biển ngày càng hoàn thiện phục vụ cho các mục đích của quốc gia.

\section{TÀI LIỆU THAM KHẢO}

Brekhovskikh, L. M., \& Lysanov, Y. P. (2003). Fundamentals of ocean acoustics ( $^{\text {rd }}$ ed.). Berlin, Germany: Springer Press.

Chengsheng, P., Liangchen, J., Ruiyan, C., \& Yuanming, D. (2012). Modeling and simulation of channel for underwater communication network. International Journal of Innovative Computing, 8(3B), 2149-2156.

Jensen, F. B., Kuperman, W. A., Porter, M. B., \& Schmidt, H. (2000). Computational ocean acoustics. Berlin, Germany: Springer Press.

John, G. P. (2001). Digital communications ( ${ }^{\text {th }}$ ed.). New York, USA: McGraw-Hill.

Johnny, R. J. (1996). Introduction to digital signal processing. New Delhi, India: Prentice-Hall of India.

Kaya, A., \& Yauchi, S. (2002). An acoustic communication system for subsea robot. Paper presented at The IEEE OCEANS Conference, USA.

Kilfoyle, D., Preisig, J., \& Baggeroer, A. (2005). Spatial modulation experiments in the underwater acoustic channel. IEEE Journal of Oceanic Engineering, 30(2), 406415 .

Kumar, R., Thakur, N., \& Thakur, V. (2013). An overview of sonar and acoustic underwater communication. International Journal of Advanced Research in Electrical, Electronics and Instrumentation Engineering, 2(5), 1997-2003.

Medwin, H., \& Clay, C. S. (1998). Fundamentals of acoustical oceanography. Massachusetts, USA: Academic Press.

Phùng, B. T. (2012). Nghiên cưu kênh thông tin duới nuớc sử dụng kỹ thuật điều chế pha QPSK. (Luận văn Thạc sĩ), Học viện Bưu chính Viễn thông, Việt Nam.

Stojanovic, M. (1996). Recent advances in high rate underwater acoustic communications. IEEE Journal of Oceanic Engineering, 21(2), 125-136. 
Stojanovic, M., Catipovic, J. A., \& Proakis, J. G. (1993). Adaptive multichannel combining and equalization for underwater acoustic communications. The Journal of the Acoustical Society of America, 94(3), 1621-1631.

Stojanovic, M. (2008). OFDM for underwater acoustic communications: Adaptive synchronization and sparse channel estimation. Paper presented at The IEEE International Conference on Acoustics, Speech and Signal Processing, USA.

Suzuki, M., Sasaki, T., \& Tsuchiya, T. (1992). Digital acoustic image transmission system for deep-sea research submersible. Paper presented at The Mastering the Oceans Through Technology Conference, USA.

Urban, H. G. (2006). Handbook of underwater acoustic engineering. The Journal of the Acoustical Society of America, 120(5), 2393-2410. 